\title{
Integrated resource management for Hybrid Optical Wireless (HOW) networks
}

\author{
Yan, Ying; Yu, Hao; Wessing, Henrik; Dittmann, Lars
}

Published in:

Conference proceedings, ChinaCOM

Link to article, DOI:

10.1109/CHINACOM.2009.5339896

Publication date:

2009

Document Version

Publisher's PDF, also known as Version of record

Link back to DTU Orbit

Citation (APA):

Yan, Y., Yu, H., Wessing, H., \& Dittmann, L. (2009). Integrated resource management for Hybrid Optical Wireless (HOW) networks. In Conference proceedings, ChinaCOM (pp. 1-5). IEEE.

https://doi.org/10.1109/CHINACOM.2009.5339896

\section{General rights}

Copyright and moral rights for the publications made accessible in the public portal are retained by the authors and/or other copyright owners and it is a condition of accessing publications that users recognise and abide by the legal requirements associated with these rights.

- Users may download and print one copy of any publication from the public portal for the purpose of private study or research.

- You may not further distribute the material or use it for any profit-making activity or commercial gain

- You may freely distribute the URL identifying the publication in the public portal

If you believe that this document breaches copyright please contact us providing details, and we will remove access to the work immediately and investigate your claim. 


\title{
Integrated Resource Management for Hybrid Optical Wireless (HOW) Networks
}

\author{
Ying Yan, Hao Yu, Henrik Wessing, Lars Dittmann \\ Department of Photonics Engineering \\ Technical University of Denmark (DTU) \\ Kgs. Lyngby, Denmark \\ \{yiya, haoyu, hewe, ladit\}@fotonik.dtu.dk
}

\begin{abstract}
Efficient utilization of available bandwidth over hybrid optical wireless networks is a critical issue, especially for multimedia applications with high data rates and stringent Quality of Service (QoS) requirements. In this paper, we propose an integrated resource management including an enhanced resource sharing scheme and an integrated admission control scheme for the hybrid optical wireless networks. It provides QoS guarantees for connections through both optical and wireless domain. Simulation results show that our proposed scheme improves QoS performances in terms of high throughput and low dropping probability.
\end{abstract}

Keywords- the hybrid orptical wireless networks; resource allocation; call admission control

\section{INTRODUCTION}

The Hybrid Optical Wireless (HOW) network is an attractive candidate for the next generation access network architectures. In the HOW networks, complementary characteristics of the optical network and wireless network are combined. In optical access network, such as Ethernet Passive Optical Network (EPON), has been deployed to satisfy bandwidth demands for broadband services, but it has a fixed architecture and limited coverage. Among various available wireless technologies, the recent Worldwide Interoperability for Microwave Access (WiMAX) technology has promised ubiquitous and high data rate connections for both fixed and mobile communication. A hybrid EPON and WiMAX network is an example of the HOW network, which is a convergence of an EPON deployed as a backhaul and multiple WiMAX networks connected as the front-end networks.

One of the major challenges for the hybrid optical wireless network is to provide end-to-end user QoS guarantees for differentiated services. Because voice and video streaming applications become ubiquitous at the remote end user sites, MAC layer protocols with strict QoS support become important. Since the optical- and wireless-access technologies are originally designed to address different issues in different network scenarios, it is difficult to solve all of the challenges by simply combining EPON and WiMAX networks together. Several issues in the MAC layer must be addressed before a highly efficient hybrid optical wireless network can be achieved, for example, the resource management issue. Since the hybrid network is under the same system management, an integrated resource managing can be accomplished, which can

This work was supported in part by the European Committee through project ALPHA (Architectures for fLexible Photonic Home and Access networks). dynamically allocate network resources based on the up-to-date wireless network and optical network conditions.

The HOW network have gained great interests in recent years. In [1], the hybrid network architectures are proposed. In [2] and [3], routing algorithm has been studied for the hybrid network and for the front-end wireless network, respectively. The scheduling algorithm is researched in [4] and [5] for the HOW network. Our previous study [6] shows a primary study of the existed network control protocol in EPON. In this paper, we propose an integrated resource management framework for the hybrid EPON and WiMAX networks. The framework consists of (1) an enhanced resource sharing scheme, which improves the existing EPON MAC protocol and (2) a novel integrated admission control algorithm, which contains a two stages admission control for the wireless domain and the optical domain, respectively.

The rest of this paper is organized as follows. Section II introduces the hybrid network model. Section III presents our proposed integrated resource management framework. In Section IV we present simulation results and performance analysis. Finally, conclusion and future work are given in Section V.

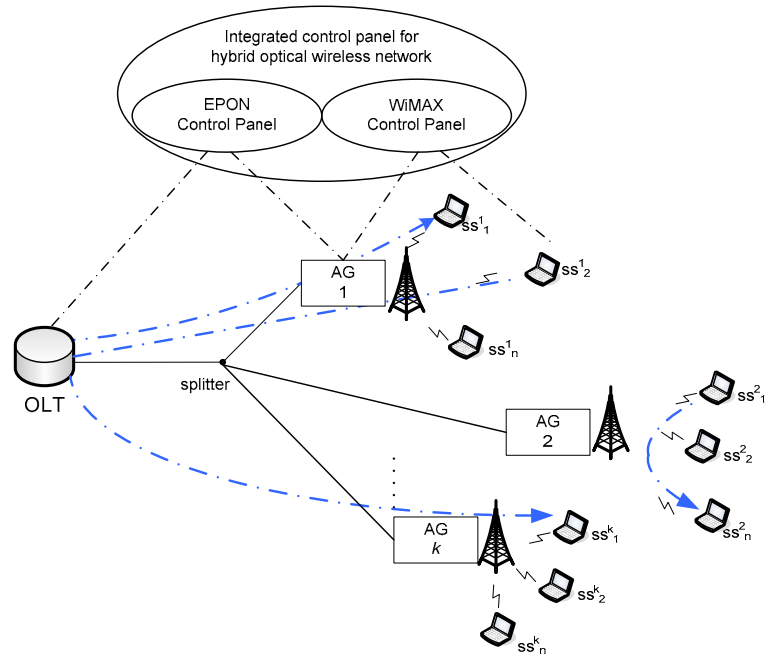

Figure 1. Network architecture of a hybrid EPON and WiMAX network 


\section{THE HYBRID NETWROK MODEL}

We consider a hybrid optical wireless network model, shown in Fig. 1. In EPON, a centralized Optical Line Terminal (OLT) communicates with multiple connected Optical Network Users (ONUs) via a passive optical splitter. In the Point-toMultipoint (PMP) WiMAX network, a Base Station (BS) manages channel allocation to Subscriber Stations (SSs) using the poll/request/grant scheme [7]. In the hybrid optical wireless network, ONU functions and BS functions are integrated into a single device, namely an Access Gateway ( $A G)$, which handles connections within the wireless network (known as the singledomain connection), or cross both EPON and WiMAX (known as the multi-domain connection). In the transport layer, an AG is the interface connecting to an OLT via fibers and to multiple SSs via wireless channels. The control planes of EPON and WiMAX can be merged into an integrated control plane, which offers optimal control solutions in admission control, scheduling, routing and signaling, according to the overall network information (e.g. link status, traffic load and congestion).

To set up a multi-domain connection in the hybrid network (a connection from an SS to the OLT), the SS initiates a request to the corresponding AG. The acceptance or rejection of a request is determined by the admission control scheme in the AG. A request can be accepted if there is enough buffer size and the QoS requirements can be satisfied. The basic idea in our enhanced admission control mechanism is to integrate information derived from both optical and wireless domain and then take the overall delay into account to provide optimal admission decisions. In order to calculate the overall delay, the network information needs to be exchanged between the optical and wireless networks. In next section, we will investigate the existing control functions in the EPON and WiMAX and propose our enhancement and improvement.

\section{Proposed InTEGRATEd RESROUCE MANAGEMENT}

\section{A. MPCP and Bandwidth Allocation Algorithms in EPON}

Two control messages, REPORT and GATE, are defined in the MultiPoint Control Protocol (MPCP) as signaling between the OLT and AGs [8]. The REPORT message is in the downstream directed to the OLT and the GATE message is in the upstream initiated from the OLT. After discovering and registering the connected AGs, the OLT sets up an entry table, which contains the AG node identification (NID), the round trip time $\left(T_{r t t}\right)$, the start transmission time $\left(t_{\text {start }}\right)$, the granted bandwidth $(B W)$. Since multiple AGs share a common optical link in the upstream direction, the OLT requires assigning the bandwidth in a manner of fairness and without conflicts. Bandwidth can be allocated statically based on the Time Division Multiple Access (TDMA) scheme or dynamically based on the resource requirement negotiation, e.g. Interleaved Polling with Adaptive Cycle Time (IPACT) [9]. In TDMA scheme, one $A G$ is granted a fixed time slot length and is polled in order. On the other hand, the IPACT scheme adopts a resource negotiation between the OLT and the AGs. The OLT polls AGs and grants time slot in a round-robin fashion. The granted time slot is determined by the requirements reported by the AG. In this paper, a cycle refers to a period in which all AGs are served and a sub-frame refers to a period, which is assigned to an AG for the uplink transmission.

\section{B. Enhanced Resource Sharing Scheme}

As mentioned earlier in this paper, the MPCP signaling discipline registers new connected ONUs. Moreover, the OLT allocates bandwidth and coordinates the uplink transmissions in order to avoid collision according to the TDMA and IPACT algorithms explained in Section III-A. After the OLT computes the start time and sub-frame length for each AG, the OLT can obtain the total transmission length $\left(T_{\text {cycle }}\right)$ for all $k$ AGs. We use $t$ to indicate the time and $T$ to indicate the duration.
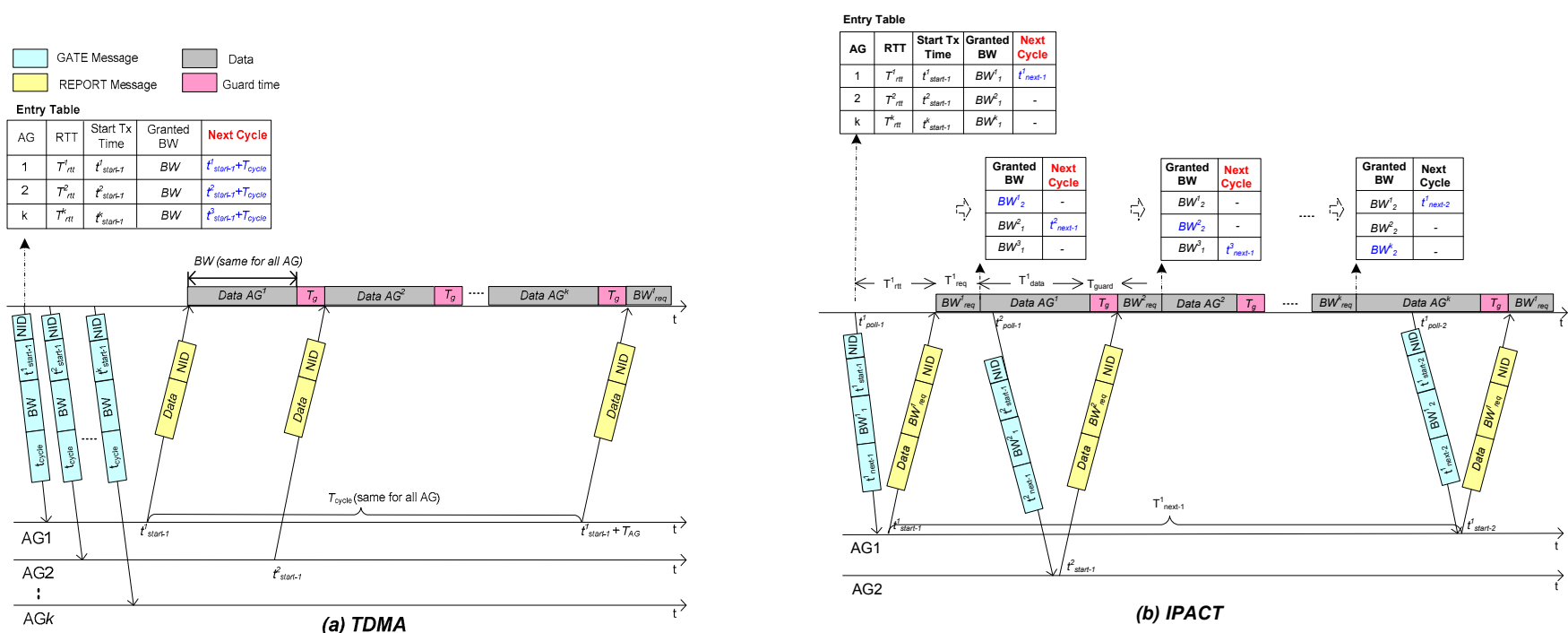

Figure 2. Modified GATE message with a new field, the next cycle time, is used with (a) TDMA scheme and (b) IPACT scheme. 


$$
T_{\text {cycle }}=\sum_{i=0}^{k-1}\left(\frac{B W^{i}}{R_{0}}+T_{g}\right)
$$

where $R_{0}$ is the transmission rate of the optical uplink and $T_{g}$ is the guard time between two successive upstream transmissions. After a period of $T_{\text {cycle }}$, an AG is polled again. The interval between two adjacent polling operations, the next cycle time $\left(t_{\text {next }}\right)$, can be calculated and added into the original GATE message as a new field.

The modified resource sharing scheme under the TDMA scheme is shown in Fig. 2a. The polling sequence of AGs is scheduled in the OLT. We assume the scheduling principle will not change. The value of granted bandwidth is fixed and same to all $k$ AGs. In other words, each AG has to wait $T_{\text {cycle }}$ as the polling wait time. The value of $t_{\text {start }}$ and $T_{\text {cycle }}$ are updated and changed once there are new AGs registered and scheduled. The expected next cycle time for the $i$-th AG is calculated in Eq. (2). The entry table is updated after the periodic discovery process. The value of $t_{\text {next }}$ for each $\mathrm{AG}$ is computed at the beginning of each cycle.

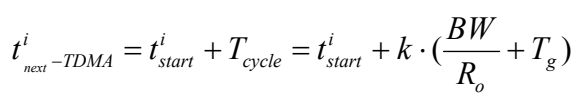

The modified resource sharing scheme under the IPACT scheme is shown in Fig. 2b. After the discovery process, the OLT fulfils the entry table with initial information of AGs. The granted bandwidth, $B W^{i}$, is assigned for the $i$-th $\mathrm{AG}$, which is used to transmit REPORT message, including data and the value of request bandwidth for next cycle $\left(B W_{\text {req }}\right)$. As explained in [9], the OLT grants AGs in an interleaved manner and distributes the length of the time slot based on the requests. For each $A G$, the start time for its upstream transmission is computed using Eq. (3). $t_{\text {poll }}^{i}$ is the time when the OLT transmits the GATE message to grant the $i^{\text {th }} \mathrm{AG}\left(\mathrm{AG}^{i}\right)$.

$$
t_{\text {start }}^{i+1}=t_{\text {grant }}^{i}+T_{r t t}^{i}+\frac{B W^{i}}{R_{o}}+T_{g}-\frac{T_{r t t}^{i+1}}{2}
$$

Because granted bandwidth is changed according to AG's request, the value of $T_{\text {cycle }}$ becomes different in cycles. Thus, the entry table is updated when the REPORT message is received. The new field, $t_{\text {next }}$, is computed and sent to the next polled AG according to the number of connected AGs and their current assigned bandwidth (Eq. 4).

$$
\begin{aligned}
& t_{\text {next-IPACT }}^{i+1}=t_{\text {start }}^{i}+T_{\text {cycle }} \\
& \quad=t_{\text {grant }}^{i}+T_{\text {rtt }}^{i}+\frac{B W^{i}}{R_{o}}+\sum_{i=0}^{k} \frac{B W^{i+1}}{R_{o}}+(k+1) \cdot T_{g}
\end{aligned}
$$

where $t_{\text {poll }}^{i}$ is the time when the OLT transmits the GATE message to the $i^{\text {th }}$ polled $\mathrm{AG}$, denoted as $A \mathrm{G}^{\mathrm{i}} . B W^{i}$ is the granted bandwidth.

\section{Integrated Optical Wireless Admission Control}

In order to support and protect the QoS of real-time traffic streams, in addition to bandwidth allocation, a call admission control scheme is required to decide whether to admit a realtime traffic stream. As we mentioned earlier, the problem of the existed resource management in hybrid optical wireless networks is lack of overall considerations of both optical and wireless network conditions. Admitting a new connection request in a WiMAX network, without considering the queuing time and polling time in the following EPON system will grant bandwidth to non-conforming traffic, e.g. to grant bandwidth to a service request, for which the delay requirements can not be met. Allocating transmission opportunities to non-conforming traffic will cause wastage of scarce bandwidth and QoS degradations to conforming traffic. In this paper, we propose an Integrated Optical Wireless Admission Control (IOW-AC) scheme, which performs rate-based AC based on the delay requirement of the new arriving traffic stream $\left(d_{\min }\right)$ and the estimated delay $\left(d_{\text {est }}\right)$.

When an SS request arrives at the $\mathrm{AG}^{\mathrm{i}}$ from the wireless domain, $A G^{i}$ is able to estimate the waiting time for the request to be served. The overall delay $\left(d_{e s t}\right)$ includes the delay time until $A G^{\mathrm{i}}$ is polled by the OLT $\left(d_{\text {polling }}\right)$, the delay time for the prior data in the same queue being served $\left(d_{\text {queueing }}\right)$, the wireless transmission delay $\left(d_{t x-\text { wireless }}\right)$ and wireless propagation delay $\left(d_{\text {prop-wireless, }}\right)$.

$$
d_{\text {est }}=d_{\text {polling }}+d_{\text {queuing }}+d_{j, t x-\text { wireless }}+d_{j, \text { prop-wireless }}
$$

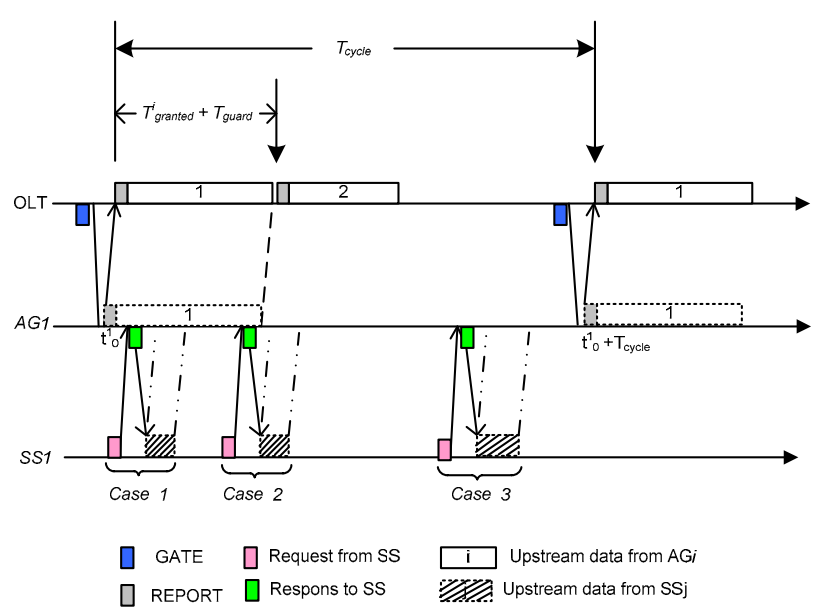

Figure 3. The upstream communication in the hybrid optical wireless network 
Where $d_{\text {queueing }}$ is calculated based on the current queue size. Because the queuing delay is not our focus, we assume the available buffer size in the polled AG is always enough. The value of $d_{t x \text {-wireless }}$ is calculated based on the request packet size and the wireless link rate. The value of $d_{\text {polling }}$ is calculated depending on the next cycle time $\left(t_{\text {next }}\right)$ and the packet arrival time $(t)$. The $t_{\text {next }}$ is computed as we explained earlier (shown in Figure 3.).

- Case 1: the request of $\mathrm{SS}^{\mathrm{j}}$ arrives when the $\mathrm{AG}^{\mathrm{i}}$ is being polled. A request can be accepted and served within the current sub-frame only if the corresponding packets can be received before the current sub-frame is finished. Thus, $d_{\text {polling }}=0$.

- Case2: the request of $\mathrm{SS}^{\mathrm{j}}$ arrives when the $\mathrm{AG}^{\mathrm{i}}$ is being polled. If the corresponding packets can not be received before the current sub-frame is finished, the request has to wait until the next cycle to be served. Thus, $d_{\text {polling }}=t_{\text {next }}$.

- Case3: the request of $\mathrm{SS}^{\mathrm{j}}$ arrives when the $\mathrm{AG}^{\mathrm{i}}$ is waiting to be polled. The earliest time for the request to get served is the next polling time. Thus, $d_{\text {polling }}=t_{\text {next }}$.

The proposed IOW-AC scheme can be realized in the AG as follows. The delay constraint with the real-time traffic streams is $d_{\text {min }}$. Since the IOW-AC is proposed to examine multi-domain connections, which transmit through both the optical and the wireless domains, in this paper we only consider requests to set up multi-domain communication. The IOW-AC performs a two-stage admission control. First, When an SS request arrives at the corresponding $\mathrm{AG}$, the transmission delay and propagation delay are calculated based on the current wireless network conditions in terms of channel condition and network utilization. The queue status is checked and queuing delay is computed according to the traffic type. Different class of traffic are separated and differentiated. Second, the packet arrival time is marked and the status of AG in the EPON is identified, being polled or waiting to be polled. The polling delay is calculated based on Eq. 2 (using TDMA scheme in EPON) or Eq. 4 (using IPACT scheme in EPON).

The advantage of this additional information is the acceptance of a new wireless connection request is decided according to not only the transmission and propagation delay in the wireless domain but also the estimated polling and queuing delay in the EPON system. Thus the overall QoS performances are guaranteed.

\section{PERFormance EVAluation}

The proposed scheme method was simulated and evaluated on the hybrid EPON and WiMAX network by OPNET simulations. The IOW-AC is compared with the normal AC. The total number of AGs is 32 , and the EPON link rate is 1 Gbps. The guard time between two adjacent transmissions on the optical uplink fibre is $5 \mu \mathrm{s}$, and the queue buffer size of an AG is 70 Mbits. WiMAX uplink data rate is up to 70 Mbps. The delay bound for the real time traffic is $75 \mathrm{~ms}$.

Fig. 4 shows the blocking ability to the non-conforming real-time traffic. IOW-AC shows better admission control

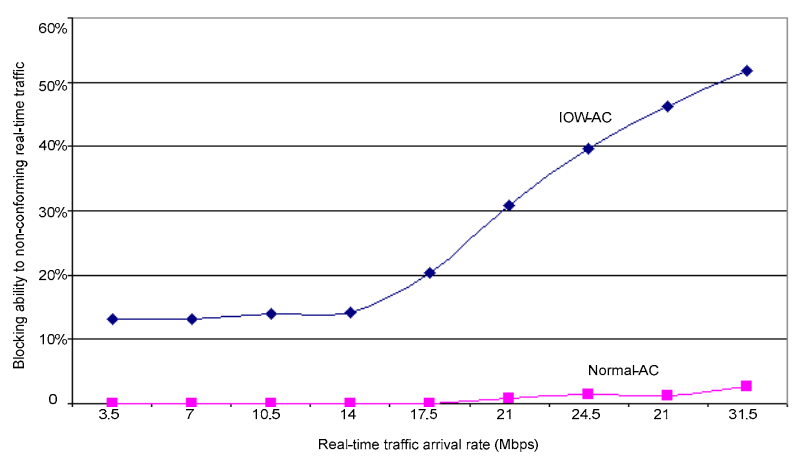

Figure 4. Blocking ability to non-conforming real-time traffic

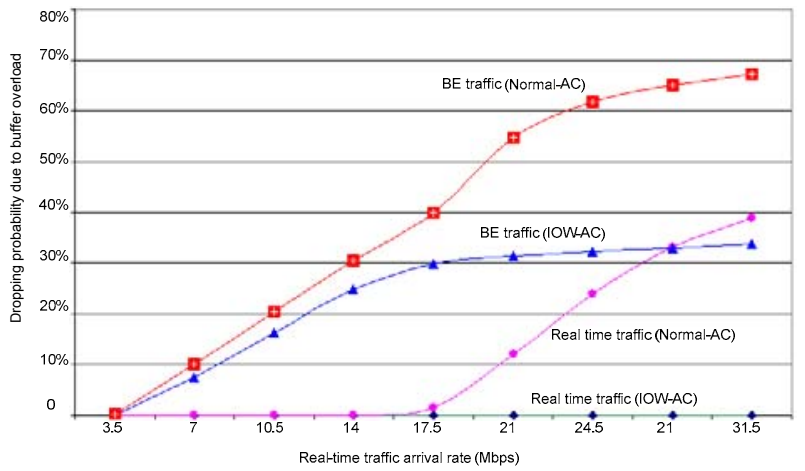

Figure 5. Packet dropping ratio due to buffer overload

ability to the real time traffic compared to the normal AC. Being aware of the next cycle time, IOW-AC is able to take the polling delay in the EPON system into account and therefore block more non-conforming traffic than the normal AC. The IOW-AC shows superior behavior especially when the realtime traffic arrival rate becomes high.

The packet dropping probability due to the buffer overload is shown in Fig. 5. Two main observations are made. First, IOW-AC scheme achieves lower dropping probability for both real time traffic and best effort (BE) traffic. This is because that IOW-AC only accepts conforming traffic and saves more bandwidth for the conforming real time traffic without any drop even under high real-time traffic rate. In addition, IOWAC serves more BE traffic instead of dropping them, which yields better bandwidth utilization.

\section{CONCLUSION}

A new scheme, integrated resource management, has been proposed in this paper for the hybrid optical wireless network. The main contributions of this paper are as follows:

a. An enhanced resource negotiation scheme, where the control messages in the existed EPON signaling protocol, MPCP, are extended with a field to carry the additional information of optical network conditions; 
b. An integrated optical wireless admission control algorithm, which makes decisions for the overall HOW network.

Proven by simulation results, our proposed scheme improves QoS performances in terms of higher network throughput and lower dropping probability for both real time traffic and best effort traffic.

\section{REFERENCES}

[1] G. Shen, R. S. Tucker and C. Chae, "Fixed mobile convergence architectures for broadband access: integration of EPON and WiMAX", IEEE Communications Magazine, pp. 44-50, August 2007.

[2] W.-T. Shaw, S.-H. Wong, N. Cheng, K. Balasubramanian, X. Zhu, M. Maier and L. G. Kazovsky, "Hybrid architecture and integrated routing in a scalable optical-wireless access network," Journal of Lightwave Technology, Vol. 25, No. 11, pp. 3443-3451.November 2007,

[3] S. Sarkar, S. Dixit and B. Mukherjee, "Hybrid wireless-optical broadband-access network (WOBAN): a review of relevant challenges", Journal of Lightwave Technology (invited paper), Vol. 25, No.11, November 2007.

[4] Y. Luo, T. Wang, S. Weinstein and M. Cvijetic., "Integrating optical and wireless services in the access network", in Proc. Optical Fiber Communication Conf., March 2006.

[5] Y. Yan, H. Yu and L. Dittmann. "Wireless channel condition aware scheduling algorithm for hybrid optical/wireless networks", in Proc. $3 r d$ International Conference on Access Networks, October 2008.

[6] Y. Yan, H. Yu, H. Wang, L. Dittmann. "Integration of EPON and WiMAX Networks: Uplink Scheduler Design", in SPIE Symposium on Asia Pacific Optical Communications, October 2008.

[7] J. G. Andrews, A. Ghosh and R. Muhamed, Fundamentals of WiMAX: understanding broadband wireless networking, Prentice Hall, 2007.

[8] Ethernet in the First Mile Alliance (EFMA), "Ethernet Passive Optical Network (EPON) Tutorial", Revision 4, 2004.

[9] G. Kramer, B. Mukherjee and G. Pesavento. "IPACT: a dynamic protocol for an Ethernet PON (EPON)," IEEE Communication Magazine, vol. 40, no. 2, pp 74-80, February 2002. 